\title{
The effects of physical and psychological stress on the performance of high- and low-anxious Ss on a difficult verbal discrimination task
}

\section{ELVIN SHEARER* and FRANK E. FULKERSON† Western Ilinois University, Macomb, Ill. 61455}

The effects of shock and ego-involving IQ-oriented instructions on the performance of high-anxious (HA) and low-anxious (LA) Ss on a difficult low-imagery verbal discrimination task were investigated. As predicted by Saltz's differential disruption hypothesis, LA Ss made significantly more errors than HA Ss in the shock condition, and HA Ss made significantly more errors in the control condition. However, no significant difference in performance was found between HA and LA Ss in the ego-involving condition. The results were interpreted as generally supportive of Saltz's view that HA and LA Ss are sensitive to different sources of stress.

Saltz $(1970,1971)$ proposed a differential disruption view as to how the Taylor manifest anxiety scale (MAS) discriminates HA and LA Ss. Saltz hypothesized that the MAS discriminates individuals on the basis of the type of stress that disrupts their performance. According to Saltz, LA Ss should perform more poorly than HA Ss on tasks where electric shock is used as the stressor, because LA Ss seem to be more susceptible to pain-induced stress than HA Ss. However, HA Ss should do worse than LA Ss on tasks where ego-involving IQ-oriented instructions and/or failure communications during learning are used, because HA Ss seem to be more sensitive to failure-induced types of stress than LA Ss. Finally, when learning tasks are very difficult, HA Ss may view the tasks themselves as threatening them with failure and, therefore, perform more poorly than LA Ss on the tasks.

The purpose of the present study was to test experimentally Saltz's predictions outlined above, using a difficult verbal discrimination (VD) list as the learning task. Several studies (e.g., Paivio \& Rowe, 1971; Ullrich $\&$ Balogh, 1972) have shown that VD lists containing all low-imagery (LI) words are very difficult to learn. The HA and LA Ss in this study learned a LI VD list under shock stress, ego-involving stress, or control conditions. It was hypothesized that (1) LA Ss would make significantly more errors than HA Ss in the shock condition, (2) HA Ss in the ego-involving condition would make significantly more errors than LA Ss given the same IQ-oriented instructions, and (3) HA Ss would make significantly more errors than LA Ss in the nonstress control condition due to the intrinsic difficulty of the task itself.

\section{METHOD}

Sixty-nine Ss (34 males and 35 females) were selected from

*Now deceased.

tReprint requests should be sent to F.E. Fulkerson, Department of Psychology, Western Illinois University, Macomb. Illinois 61455 . introductory psychology classes on the basis of scores they achieved on the MAS. The HA and LA Ss were those whose scores fell in the upper 25\% (i.e., scores from 25 to 40 ) and the lower 25\% (i.e., scores from 4 to 14), respectively, of all Ss taking the MAS. The Ss whose scores were in the desired range were given an opportunity to volunteer for this experiment to receive extra credit for their introductory classes. The MAS was given under the description of a biographical inventory scale to approximately 300 introductory psychology students, who received the 50-item MAS with 40 filler items from a biographical inventory scale of the Minnesota Multiphasic Personality Inventory. A double-blind procedure was used to assign the HA and LA volunteer $S s$ to the experimental conditions. The design was a 2 by 3 by 2 factorial, with manifest anxiety level (HA or LA), type of stress (shock, ego-involving, or control), and sex of $S$ as the between-group experimental and subject variables. Only approximately equal numbers of Ss served in each experimental condition, because an insufficient number of HA Ss volunteered to participate in the experiment.

The Ss in the shock stress group were told that they would receive unpleasant shocks during the experiment. These Ss were then given a sample shock before beginning the VD learning and were told that they would receive further shocks throughout the experiment. Actually, the Ss received no additional shocks. The shocking device used was a constant-current power source producing a $2.5-\mathrm{mA}$ shock. The shock was administered through two large wrist electrodes attached to the $S$ throughout the experiment. The duration of the sample shock was approximately $1 / 2 \mathrm{sec}$.

The Ss in the ego-involving stress group were told that performance on this particular VD task had been found to be highly correlated with intelligence. "The higher your intelligence level," Ss were told, "the fewer mistakes you will make on the verbal discrimination task." The Ss in the control group were told that the $\mathrm{E}$ was interested in the difficulty of the VD task and that their performances would be used to evaluate other students who would learn the VD list in later studies.

All Ss learned a 16-pair LI VD list, which had been chosen from the norms of Paivio, Yuille, \& Madigan (1968). The list had a mean imagery value of 3.25 , a mean meaningfulness value of 5.5 , and a mean frequency value of 22.5. The VD list was presented individually to each $S$ on a Lafayette memory drum in a standard study-test manner for eight alternating study and test trials. On the study trials, each pair of "right" and "wrong" words appeared in juxtaposition for a 2-sec presentation, with the "right" word underlined. After a 4-sec intertrial interval, a test trial was presented in the same manner as the study trial, except the "right" word of each pair was not underlined. Eight different random orders for study and test trials were used to control for serial effects, and spatial position of the "right" and "wrong" items was counterbalanced. The Ss were given standard VD instructions, including instructions to begin guessing on the first test trial. After completion of the learning task, all Ss were debriefed.

\section{RESULTS AND DISCUSSION}

A 2 by 3 by 2 unweighted means between-group analysis of variance for unequal ns was performed on the VD error data to determine the significance of the results. Only the Stress by Anxiety interaction was found to be significant, $F(2,58)=6.25, p<.005$. No other main or interaction effects were significant.

Figure 1 presents the mean number of errors made by 


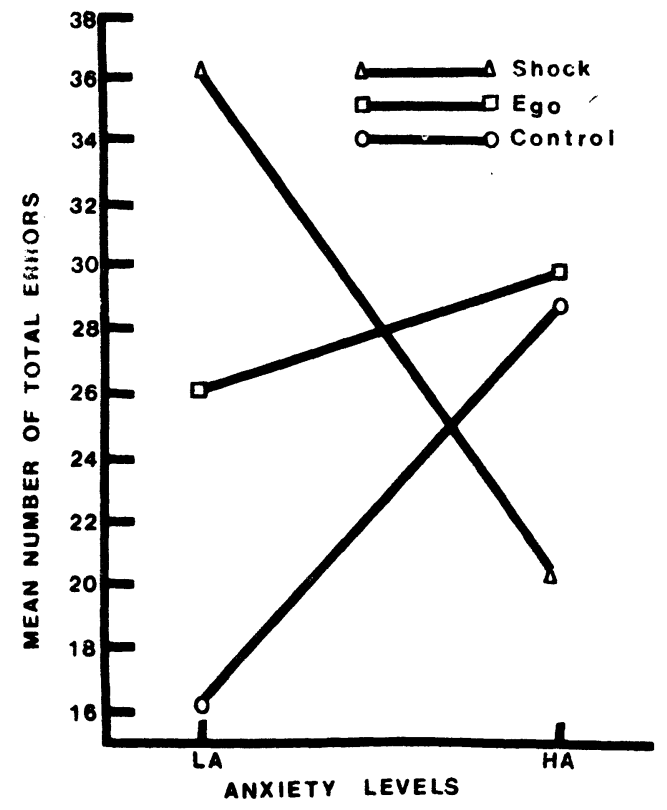

Fig. 1. Mean number of total errors as a function of anxiety level and stress condition.

HA and LA Ss in the different stress conditions. Duncan's test for multiple comparisons for nearly equal ns was used to determine the significance levels between the individual means. It was found that LA Ss made significantly more errors than HA Ss when shock was used as the external stressor $(p<.05)$. Also, HA Ss made significantly more errors than LA Ss in the control condition $(p<.05)$, where the only stressor was the difficulty of the task itself. However, no significant difference was found between the performances of the HA and LA Ss in the ego-involving condition, where the IQ-oriented instructions were used. In addition, the LA control group performed significantly better than both the LA shock group $(p<.01)$ and the HA ego-involving group $(p<.05)$. No other comparisons reached significance.

Thus, as predicted, LA shocked Ss performed more poorly than HA shocked Ss, and HA Ss made more errors than LA Ss on the difficult task when neither shock nor high-motivation instructions were used. These two findings give support to Saltz's disruption view of how HA and LA Ss are discriminated by the MAS. However, no difference between HA and LA Ss under ego-involving instructions was shown. If HA Ss become disrupted by instructions that induce the threat of failure, then HA Ss should have performed more poorly than LA Ss under IQ-oriented instructions. The instructions themselves in the present study may have been insufficient to produce the threat of failure. Sarason \& Sarason (1957) gave Ss high-motivation instructions or failure statements and found that Ss given failure statements during learning showed an immediate decline in performance. Ss given only high-motivation instructions did not show the immediate disruption effect. Sarason \& Palola (1960) gave MAS HA and LA Ss difficult or easy anagram tasks and high- or low-motivation instructions. The results showed that HA Ss had more trouble with the difficult anagrams than LA Ss, but no difference was found between HA and LA Ss under high- or low-motivation instructions. Also, only a trend was reported for the three-way interaction, with HA Ss performing slightly worse than LA Ss when given both a difficult task and high-motivation instructions. Sarason's studies seem to indicate that producing a failure effect by using only high-motivation instructions may be difficult and may not appear if other statements forecasting failure (i.e., statements emphasizing Ss' inferior performance during learning) are not used.

Sarason (1957) also suggested that sex of Ss and E may interact with the anxiety level of the Ss. This would seem particularly likely when instructions are given to the Ss that are ego-involving. In the present study, a male E told both male and female Ss that the VD task was a measure of intelligence. The mean number of errors in the ego-involving condition suggested that perhaps HA females $(\bar{X}=32.5)$ were more disrupted by these instructions than were HA males $(\bar{X}=21.6)$, LA females $(\bar{X}=25.3)$, or LA males $(\bar{X}=26.7)$. Thus, the failure of this study to produce a significant difference between HA and LA Ss in the ego-involving condition may have been due to the action of various confounding variables. It seems likely that to produce this disruption effect many factors need to be carefully controlled. Among these are the strength of the verbal instructions themselves (i.e., whether the instructions are believably worded and given), the sex of both the $S$ and the $E$, and whether statements reinforcing the threat of failure are given during the learning task.

Nevertheless, the overall findings of this study seem to support Saltz's position that the MAS is not discriminating Ss on an anxiety continuum but that Ss' scores on the scale are related to the type of stress that most disrupts their performance. However, it appears that further studies are needed which might include easy as well as difficult tasks and failure statements as well as IQ-oriented instructions in order to better assess Saltz's hypothesis.

\section{REFERENCES}

Paivio, A., \& Rowe, E. J. Intrapair imagery effects in verbal discrimination and incidental associative learning. Canadian Journal of Psychology, 1971, 25, 302-312.

Paivio, A., Yuille, J. C., \& Madigan, S. A. Concreteness, imagery, and meaningfulness values for $\mathbf{9 2 5}$ nouns. Journal of Experimental Psychology Monograph Supplement, 1968, 76(1, Pt. 2).

Saltz, E. Manifest anxiety: Have we misread the data? Psychological Review, 1970, 77, 568-573.

Saltz, E. The cognitive basis of human learning. Homewood, Ill: Dorsey, 1971.

Sarason, I. G. The effect of anxiety and two kinds of motivating instructions on verbal learning. Journal of Abnormal \& Social Psychology, 1957, 54, 166-171.

Sarason, I. G, \& Palola, E. G. The relationship of test and general antiety, difficulty of task, and experimental instructions to performance. Journal of Experimental Psychology, 1960, 59, 185-191.

Sarason, I. G., \& Sarason, B. R. Effects of motivating instruction and reports of failure on verbal learning. A merican Journal of Psychology, 1957, 70, 92-96.

Ullrich, J. R., \& Balogh, B. A. Imagery and meaningfulness of right and wrong items in verbal discrimination learning. Psychonomic Science, 1972, 29, 68-70.

(Received for publication January 29, 1973.) 